\title{
Fibras vegetais utilizadas no artesanato comercializado em Boa Vista, Roraima $^{1}$
}

\author{
Andréia Silva Flores ${ }^{2} \&$ Divina da Silva Lima ${ }^{2}$
}

1. Tema de trabalho de conclusão de curso de Ciências Biológicas das Faculdades Cathedral, Boa Vista, Roraima.

2. Instituto de Amparo de Ciência, Tecnologia e Inovação de Roraima. Museu Integrado de Roraima. Herbário MIRR. Av. Brigadeiro Eduardo Gomes s.n., Parque Anauá, Bairro Aeroporto, Boa Vista, Roraima CEP 69305-010. Autor para correspondência: andreiasflores@gmail.com

Recebido em : 10/01/2013. Aceito em: 18/02/2013.

RESUMO

As fibras vegetais são utilizadas de diversas formas e maneiras, gerando emprego renda e cidadania. O artesanato é uma atividade que gera renda com base no manejo responsável das matérias primas. O objetivo principal deste estudo foi analisar o artesanato com fibras vegetais comercializado no município de Boa Vista, Roraima, visando identificar as principais espécies e partes vegetais utilizadas e como são elaboradas as peças artesanais. Os dados sobre as espécies vegetais, os utensílios fabricados e sua utilização, foram obtidos através de entrevistas semiestruturadas com os comerciantes e vendedores. Foram identificadas sete espécies, distribuídas entre seis gêneros e quatro famílias. A família Arecaceae apresentou três espécies: Astrocaryum aculeatum G. Mey., Mauritia flexuosa L.f. e Desmoncus polyacanthos Mart.; Marantaceae registrou duas espécies: Ischnosiphon obliquus (Rudge) Körn. e Ischnosiphon arouma (Aubl.) Körn., enquanto que Araceae apresentou apenas a espécie Heteropsis spruceana Schott. A família Sapindaceae registrou a utilização de uma espécie do gênero Serjania. Os principais órgãos das plantas utilizadas são as raízes, folhas e caules. As fibras são comercializadas na forma de jóias, móveis, objetos decorativos e em sacolas com diferentes pesos.

PALAVRAS CHAVE: Botânica; Arecaceae; Amazônia; Brasil.

\section{ABSTRACT}

Vegetable fibers are used in various ways and manners, income generating employment and citizenship. The handicraft is an activity that generates income based on responsible management of raw materials. The main objective of this study was to analyze the craft with vegetable fibers sold in the city of Boa Vista, Roraima, to identify the main species and plant parts used and how the handicrafts are made. The data concerning about the plant species, and the vessels manufactured and its use were obtained through semi-structured interview. Seven species were identified, distributed among six genera and four families. The family Arecaceae had three species: Astrocaryum aculeatum G. Mey., Mauritia flexuosa L.f. e Desmoncus polyacanthos Mart.; Marantaceae recorded two species: Ischnosiphon obliquus (Rudge) Körn. e Ischnosiphon arouma (Aubl.) Körn, and one of the Araceae: Heteropsis spruceana Schott. The family Sapindaceae recorded the use of a species of the genus Serjania. The main organs of the plants used are the roots, leaves and stem. The fibers are marketed in the form of jewelry, furniture, decorative objects and bags of different weights.

KEY WORDS: Botany; Arecaceae; Amazon; Brazil.

\section{INTRODUÇÃO}

As fibras vegetais são utilizadas de diversas formas e maneiras, gerando emprego renda e cidadania. $\mathrm{O}$ artesanato é uma atividade que gera renda com base no manejo responsável das matérias primas. A partir do conhecimento básico sobre como são realizadas estas atividades se podem diagnosticar os principais problemas encontrados para a ampliação desta atividade e a procura da solução destes problemas. As espécies vegetais fibrosas ocupam papel de destaque no cotidiano das comunidades tradicionais amazônicas ao lado das espécies medicinais, alimentícias e madeireiras.

Na região Norte há muitos estudos sobre este tipo de atividade, predominantemente realizados nos estados do Pará e Amazonas (Durigan \& Castilho 2001; Fagury 2005; Nakazono 2006; Oliveira et al. 2006, entre outros). Estes estudos podem auxiliar o artesão e a economia de certas regiões a oferecer subsídios para estudos mais avançados que possibilitem avaliar as potencialidades econômicas dessas espécies vegetais (Pastore Junior \& Borges 1995; Gonçalves et al. 2012).

Em Roraima, não há publicado nenhum estudo sobre o artesanato com fibras vegetais. $\mathrm{O}$ presente trabalho tem como objetivo caracterizar o artesanato com fibras vegetais comercializado no município de Boa Vista, Roraima. Assim, pretende-se também colaborar com o conhecimento tradicional sobre o uso da flora em atividades sustentáveis e subsidiar ações de políticas públicas que visem à ampliação deste tipo de atividade geradora de renda com baixos impactos na natureza.

\section{MATERIAL E MÉTODOS}

Área de estudo: O município de Boa Vista 
apresenta área territorial de 5.687,06 $\mathrm{Km}^{2}$ que corresponde a $2,54 \%$ do território de Roraima. O município localiza-se a centro leste do Estado de Roraima, situado nas coordenadas geográficas $60^{\circ} 40^{\prime} 24^{\prime \prime} \mathrm{W}$ e $02^{\circ} 49^{\prime} 11^{\prime \prime} \mathrm{N}$, com altitude de $85 \mathrm{msm}$. O clima é tropical úmido do tipo "A", do subtipo AW, quente e úmido, com estação chuvosa no verão. Apresenta precipitação média de 1.750 milímetros anuais. A temperatura média anual é de $27,4^{\circ} \mathrm{C}$. A média da umidade relativa do ar é de $75 \%$. A estrutura produtiva do município está assentada basicamente no setor terciário. A geração de emprego é realizada, sobretudo, pelo setor público, que abriga o maior contingente, seguido do setor comercial. A produção primária é essencialmente baseada na pecuária e na cultura do arroz sequeiro, soja e milho. $\mathrm{O}$ extrativismo no município é insignificante (SEPLAN-RR 2010).

Coleta de dados: Este estudo foi realizado com a coleta de informações sobre a produção dos artigos de artesanato em estabelecimentos que comercializam estes produtos e na revisão de literatura. Também foram anotadas as informações sobre $\mathrm{o}$ produto em etiquetas anexadas a estes.

A comercialização de artesanatos é realizada predominantemente no Centro de Boa Vista, em lojas especializadas, que compram diretamente os produtos dos artesãos.

Foram entrevistados comerciantes e vendedores da cidade de Boa Vista, em locais de venda de artesanato em fibras vegetais. Os dados sobre as espécies vegetais, os utensílios fabricados e sua utilização, foram obtidos por meio de entrevistas semiestruturadas. Imagens foram obtidas com o auxílio de máquina digital fotográfica.

A entrevista apresentou questões semiestruturadas, nos quais foram levantadas as informações sobre a planta utilizada para o artesanato (nome popular, órgão da planta utilizado, forma de vida, locais de coleta) e dados do objeto comercializado (produtos confeccionados, principais técnicas e utilização do objeto).

Após as informações prestadas, as espécies vegetais foram fotografadas e coletadas. As plantas coletadas foram identificadas e incorporadas ao acervo do herbário MIRR (Herbário do Museu Integrado de Roraima).

A identificação foi efetuada utilizando literatura especializada. Além da coleta, foram efetuados registros fotográficos das espécies, da forma de obtenção da matéria prima, do processo de confecção dos objetos.

\section{RESULTADOS E DISCUSSÃO}

\section{Principais espécies utilizadas no artesanato em fibras de Boa Vista}

Foi registrada a utilização de sete espécies, distribuídas entre seis gêneros e quatro famílias (Tabela 1): Astrocaryum aculeatum G. Mey, Mauritia flexuosa L.f., Desmoncus polyacanthos Mart. (Arecaceae), Ischnosiphon obliquus (Rudge) Körn., Ischnosiphon arouma (Aubl.) Körn. (Marantaceae), Heteropsis spruceana Schott (Araceae) e Serjania sp. (Sapindaceae).

As principais procedências dessas fibras para a produção do artesanato foram as regiões noroeste e sudeste de Roraima. Estas espécies apresentam sua distribuição geográfica natural centrada principalmente na região amazônica, exceto por Mauritia flexuosa e Desmancus polyacanthos que apresentam distribuição geográfica mais ampla ocorrendo também nas regiões sudeste e nordeste.

Observou-se que a forma de vida herbácea (incluindo as trepadeiras) ocupa o primeiro lugar com relação à forma de vida nas espécies de "arumã" (Ischnosiphon arouma e Ischnosiphon obliquus), "cipó titica" (Heteropsis spruceana) e "cipó timbó-açú" (Serjania sp.). O porte arbóreo foi registrado nas espécies de palmeiras: "buriti" (Mauritia flexuosa), "Tucumã" (Astrocaryum aculeatum) e "Jacitara" (Desmoncus polyacanthos).

A família Arecaceae apresentou um maior número de espécies utilizadas para a produção de artesanatos, seguido de Maranthaceae. Observa-se que na maioria dos estudos sobre artesanato em fibras na Amazônia, Arecaceae, é a família mais representativa. Porém, nestes estudos é relatada a utilização de muitas outras espécies e gêneros da família. Além de Arecaceae e Maranthaceae também são relatados nestes estudos a utilização de espécies das famílias Cyclanthaceae, Bignoniaceae, Dilleniaceae, Bombacaceae, Poaceae, Cyperaceae e Combretaceae (Potiguara et al. 1987; Oliveira et al. 2006), as quais não foram registrados no presente estudo. 
Tabela 1. Espécies de fibras vegetais utilizadas no artesanato do município de Boa Vista.

\begin{tabular}{|l|c|l|}
\hline Táxon & Nome Vulgar & Órgãos usados \\
\hline Heteropsis spruceana & Cipó titica & Raízes aéreas \\
\hline Mauritia flexuosa & Buriti & Folhas jovens \\
\hline Astrocaryum aculeatum & Tucumã & Pecíolo/raque \\
\hline Desmoncus polyacanthos & Jacitara & Caule \\
\hline Ischnosiphon arouma & Arumã & Haste caulinar \\
\hline Ischnosiphon obliquus & Guarimã & Haste caulinar \\
\hline Serjania sp. & Timbó-açu & Raízes aéreas \\
\hline
\end{tabular}

Órgãos vegetais utilizados no artesanato com fibras

Poucos comerciantes conheciam as técnicas básicas do preparo dos materiais, quando visitavam os artesãos para a compra dos produtos.

Em Astrocaryum aculeatum ("Tucumã") os principais órgãos utilizados da planta são a raque foliar e o pecíolo da folha, de onde a fibra é extraída. Em Mauritia flexuosa "buriti" os órgãos utilizados são as folhas jovens. A coleta é feita por mulheres que coletam das palmeiras pequenas, a coleta em palmeiras altas é feita exclusivamente por homens que usam uma vara com uma foice na ponta ou sobem nos buritizeiros. Na coleta é utilizado o corte chamado de bico de gaita para que brote novamente. $\mathrm{O}$ beneficiamento da matéria-prima atividade envolve uma série de etapas, que são realizadas geralmente por mulheres, que para a maior parte delas representa uma das únicas fontes de renda. O órgão utilizado em Desmoncus polyacanthos "Jacitara" é o caule, que após a retirada das bainhas e as folhas completas, apresenta a parte interna do caule, do qual se extrai as talas para fabricação de peneiras e sacolas. Esse material tem certa preferência por parte dos artesãos por apresentar maior resistência e durabilidade que as demais espécies.

Os órgãos utilizados em Serjania sp "timbóaçú" e em Heteropsis spruceana "cipó-titica" são as raízes aéreas. Durante a coleta, são deixadas algumas das raízes para que a planta regenere, os cipós são puxados das castanheiras em que estão apoiados, os nós e as cascas são desprezados no local de coleta, o cipó é levado para casa, logo são separados e enumerados em um aparelho chamado de enumerador de cipó, que possui furos que variam no seu tamanho. Os cipós são tingidos com a casca da castanheira e algumas vezes com tintura de tecido, as confecções com cipós de cores naturais possuem maior facilidade de venda.

Os órgãos utilizados em Ischnosiphon obliquus "guarimã", e Ischnosiphon arouma "arumã" são os talos da haste caulinar. A coleta é sempre feita com trabalho manual em qualquer período do ano. $\mathrm{O}$ artesanato é feito com as talas, da haste caulinar da arumã. As talas são cortadas de indivíduos adultos com cerca de 2 a 5 metros de altura ou mais. O corte é feito com facas afiadas e com pequenos facões. Após o corte as talas são transportadas para casa. Todo preparo das talas é feito na área de casa, onde é desfiada para a retirada da fibra da arumã, em seguida são separadas em feixes, o trabalho é sempre manual. Os indivíduos jovens e as folhas não são utilizados.

\section{Principais produtos artesanais comercializados a partir da fibra vegetal em Boa Vista.}

Vários produtos artesanais são confeccionados a partir da fibra destas espécies e comercializados em Boa Vista (Fig. 1 A-G). As fibras das Arecaceas são utilizadas na produção de cestarias, saias, brincos, sutiãs, pulseiras, tornozeleiras, tapetes, entre outras peças. Esteiras, cestarias e abanos são produzidos utilizando as fibras do "tucumã". Com a fibra da "jacitara" são produzidos as peneiras, sacolas e cestos. Com a fibra retirada do "buriti" são produzidos diversos produtos artesanais, sendo uma das fibras mais utilizadas devido a facilidade da localização e freqüência dos indivíduos nos locais de coleta.

Em trabalho realizado por Santos \& Ferreira (2012) foram registrados 21 tipos de utilização da fibra de "buriti", popularmente conhecido como "miriti" no Pará. Além da confecção dos produtos artesanais mencionados acima, os autores indicam a grande importância da fibra desta espécie para produção de brinquedos, tipiti, cordas, paredes e janelas de casas.

Com a fibra do "cipó-titica" são produzidos 

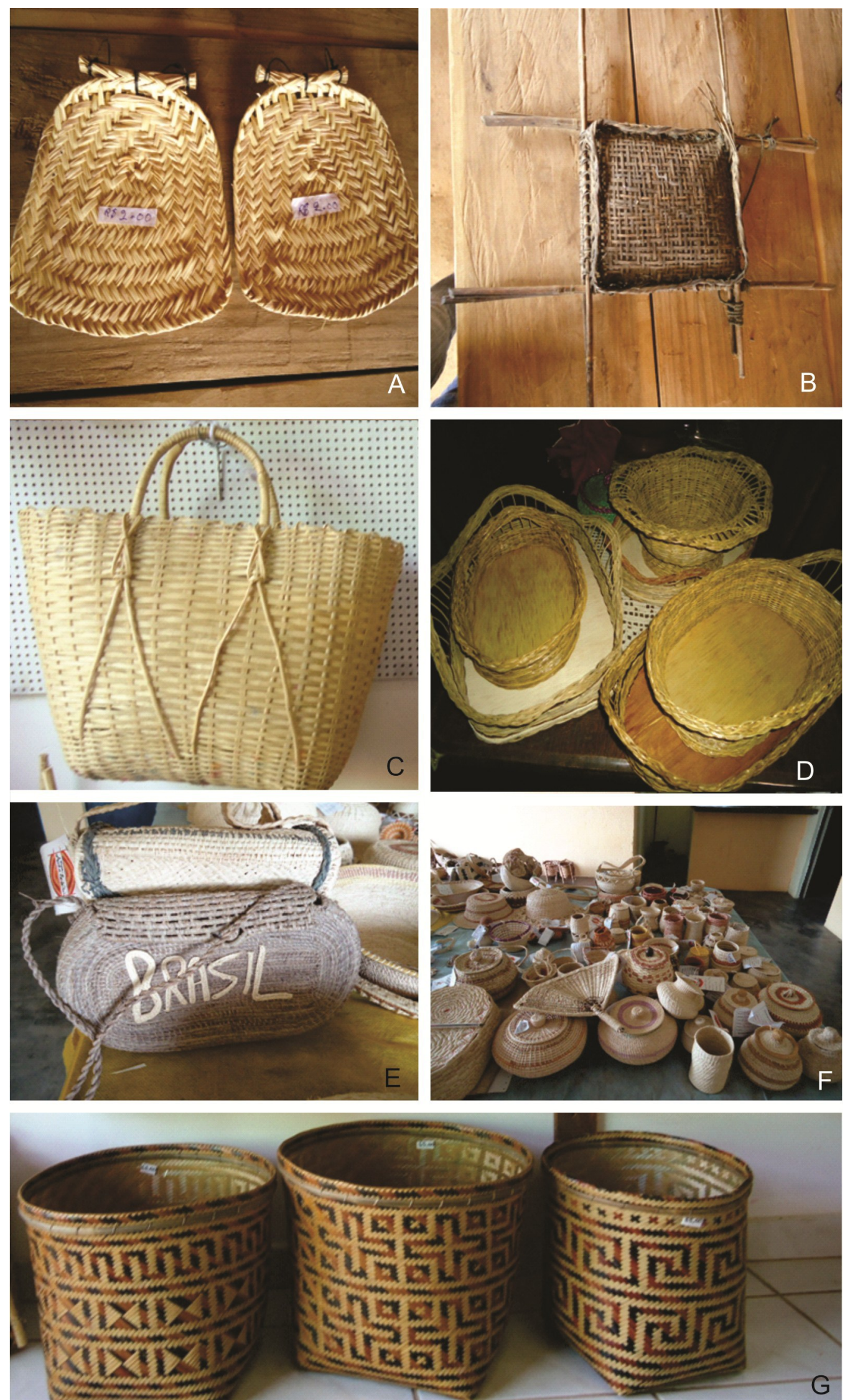

Figura 1. Produtos artesanais confeccionados com fibra vegetal comercializados em Boa vista, Roraima. A. Abano de fibra de Astrocaryum aculeatum (tucumã); B. Peneira de fibra de Desmoncus polyacanthos (jacitara); C. Sacola de fibra de Mauritia flexuosa (buriti); D. Cestas de fibra de Heteropsis spruceana (cipó titica);E. Bolsa elaborada com a fibra do Mauritia flexuosa (buriti); F. Produtos produzidos com fibra de Mauritia flexuosa (buriti); G. Cestarias de fibra de Ischnosiphon arouma (arumã) 
cestarias, mesas, estantes, sofás, cadeiras, vasos, vassouras e outros tipos de produtos. A fibra do "timbó-açúu" é utilizada para a produção de vassouras, sofás baús, sapateiras e outros. As raízes aéreas das espécies de Heteropsis são muito procuradas para confecção de peças artesanais pelos habitantes da região amazônica. Segundo Durigan \& Castilho (2004) a grande flexibilidade das raízes aéreas das espécies do gênero permitem o uso para amarras e confecção de cestos, vassouras, móveis e artesanato em geral.

A fibra extraída do "arumã" é utilizada para confeccionar cestos, esteiras, balaios, peneiras, quibanos e outros produtos decorativos, de uso pessoal e doméstico e com as fibras do "guarimã" são produzidos as cestaria, portas jóias e porta canetas. Em ambas as espécies a fibra extraída também são utilizadas na confecção de porta garrafas, pequenos cestos para pães, bolsas, apoios para panelas.

\section{CONCLUSÃO}

Foram encontradas sete espécies no comércio artesanal de Boa Vista e estão distribuídas entre seis gêneros e quatro famílias, sendo que Arecaceae apresentou o maior número de espécies. Estudos na Amazônia brasileira apontam esta família como a principal fonte de fibra vegetal. Entretanto, várias outras espécies fornecedoras de fibras também poderiam estar sendo utilizadas para a confecção de artesanato.

Os principais órgãos utilizados da planta são a raque foliar, pecíolo, folhas jovens e caule em espécies de Arecaceae, raízes aéreas em Araceae e Sapindaceae e haste caulinar nas espécies de Maranthaceae.

As fibras são utilizadas para a fabricação de diversos objetos decorativos, principalmente nas cestarias. Uma recomendação de manejo dentro de um programa de organização e conscientização dos coletores pode ser adotada em Roraima, além do desenvolvimento de estudos sobre a biologia das espécies exploradas.

\section{REFERÊNCIAS BIBLIOGRÁFICAS}

Durigan, C.C. \& Castilho, C.V. 2004. O extrativismo de cipós (Heteropsis spp., Araceae) no Parque Nacional do Jaú. In: Borges, S.H.; Iwanaga, S; Durigan, C.C. \& Pinheiro, M.R. (Org.). Janelas para a Biodiversidade no Parque Nacional do Jaú: uma estratégia para o estudo da biodiversidade na Amazônia. Fundação Vitória Amazônica, Manaus. p. 231-244.

Fagury, R V G. 2005. Avaliação de fibras naturais para a fabricação de Compósitos: açaí, coco e juta. (Dissertação de Mestrado), Universidade Federal do Pará Centro Tecnológico Programa de PósGraduação em Engenharia Mecânica, Belém.

Gonçalves, D.C.M., Gama, J.R.V., Oliveira, F.A., Oliveira Junior, R.C., Araújo, G.C. \& Almeida, L.S. 2012. Aspectos Mercadológicos dos Produtos não Madeireiros na Economia de Santarém-Pará, Brasil. Floresta e Ambiente 19(1): 9-16.

Nakazono, E.M. \& Piedade, M.T.F. 2004. Biologia e ecologia do arumã, Ischnosiphon polyphyllus (Marantaceae), no arquipélago de Anavilhanas, Rio Negro, Amazônia Central. Revista Brasileira de Botânica v.27 (3): 421-428.

Oliveira, J.; Potiguara, R.C.V.; Lobato, L.C.B. 2006. Fibras vegetais utilizadas na pesca artesanal na microrregião do Salgado, Pará. Boletim do Museu Paraense Emílio Goeldi, série Ciências Humanas, Belém, v. 1 (2): 113-127.

Pastore Junior F. \& Borges, V. 1995. Extração florestal não-madeireira na Amazônia: Armazenamento $e$ comercialização. ITTO, FUNATURA, IBAMA, LATEQ-UnB; 73 p.

Potiguara, R. C. V.; Almeida S.S.; Oliveira, J; Lins, A.L.F.A. \& Lobato, L.C.B. 1987. Plantas fibrosas I: levantamento botânico na microrregião do Salgado (Pará-Brasil). Boletim do Museu Paraense Emílio Goeldi, série Botânica, v. 3 (2): 279-301.

Santos, R.S. \& Ferreira, M.C. 2012. Estudo etnobotânico de Mauritia flexuosa L. f. (Arecaceae) em comunidades ribeirinhas do Município de Abaetetuba, Pará, Brasil. Acta Amazonica, v. 42(1): $1-10$.

SEPLAN-Secretaria de Estado do Planejamento e Desenvolvimento de Roraima. 2010. Informações Socioeconômicas do Município de Boa Vista - RR. $1^{\mathrm{a}}$ edição.Boa Vista: CGEES/SEPLAN - RR.68p. 